\title{
Electrical conduction in the system $\mathrm{Sr}_{0 \cdot 90} \mathrm{La}_{0 \cdot 10} \mathrm{Ti}_{0 \cdot 90} \mathrm{M}_{0 \cdot 10}^{\prime} \mathrm{O}_{3}$ $\left(\mathrm{M}^{\prime}=\mathrm{Co}, \mathrm{Ni}\right.$ or $\left.\mathrm{Cr}\right)$
}

\author{
H S TEWARI and R K SINGH* \\ Department of Physies, Barkatullah University, Bhopal 462026, India \\ * Institute of Physics, Ciuru Ghasidas University, Bilaspur 495009 , India \\ MS received 24 June 1996
}

\begin{abstract}
We have successfully synthesized the system $\mathrm{Sr}_{1-x} \mathrm{La}_{x} \mathrm{Ti}_{1-x} \mathrm{M}_{x}^{\prime} \mathrm{O}_{3}$ where $\mathbf{M}^{\prime}=\mathrm{Cr}$, Ni and $\mathrm{Co}$ by using conventional solid stite ceramic method. Powder X-ray diffraction patterns of the different compositions show the formation of single phase materials. Measurement of $\mathrm{AC}$ conductivity as a function of frequency at different temperatures in the range $300-550 \mathrm{~K}$ show that conduction in these compositions occurs due to hopping of charge carriers between locaized transition metal ion sites.
\end{abstract}

Keywords. Peruvskite; substitutions: hopping conduction.

\section{Introduction}

Alkaline earth iitanates exhibit interesting dielectric properties with high resistivity and are useful in electronic applications. In this class of materials, properties are governed by distortion in lattice i.e. change in lattice parameters and symmetry. In case of oxides of transition metal ions with lanthanam, electrical properties are governed by the valence state and the spin state of the transition metal ions. The properties of these materiais can further be modified and exploited for technical applications by compositional modifications and by changing different processing parameters (Buchanan 1986). For the last few years, we are studying the dielectric and electrical properties of valence compensated solid solutions of these oxides. For the system $\mathrm{M}_{1-x} \mathrm{La}_{x} \mathrm{Ti}_{1} \cdot \mathrm{Co}_{x} \mathrm{O}_{3}(\mathrm{M}=\mathrm{Pb}, \mathrm{Ba}, \mathrm{Sr}$ and $\mathrm{Ca}$, studies on electrical behaviour show that conductivity continuously increases with increase in $x$. Since other ions have inert gas configuration, it is concluded that cobalt ions in these systems play an important role in electrical conduction behaviour (Parkash e' al 1987, 1989a, b, 1990). In order to study the effect of transition rnetal ions on electrical properties of valence compensated perovskite oxides, a typical composition with different transition metal ions $\mathrm{Sr}_{0.90} \mathrm{La}_{0 \cdot 10} \mathrm{Ti}_{0 \cdot 90} \mathrm{M}_{0 \cdot 10}^{\prime} \mathrm{O}_{3}\left(\mathrm{M}^{\prime}=\mathrm{Co}\right.$. Ni and $\left.\mathrm{Cr}\right)$ prepared under identical conditions, was studied. This represent the solid solutions between $\mathrm{SrTiO}_{3}$ and $\mathrm{LaCoO}_{3}, \mathrm{LaNiO}_{3}$ or $\mathrm{LaCrO}_{3}$.

\section{Experimental}

The investigated samples were prepared by conventional solid state ceramic method by mixing appropriate quantities of strontium carbonate, titanium dioxide, lanthanum oxalate, cobalt oxalate, basic nickel carbonate and chromium oxide, all having purity better than $99.5 \%$ in an agate mortar using acetone as a grinding medium. The mixed,

\footnotetext{
*Author for correspondence
} 
Table 1. Values of lattice parameter, a $(\AA)$, activation energies, $E_{\mathrm{a}}$ and $E_{\mathrm{b}}(\mathrm{eV})$ and hopping energy, $W_{\mathrm{H}}(\mathrm{eV})$ for different compositions in the system, $\mathrm{Sr}_{0.90} \mathrm{La}_{0.10} \mathrm{Ti}_{0.90} \mathrm{M}_{0.10}^{\prime} \mathrm{O}_{3}\left(\mathrm{M}^{\prime}=\mathrm{Co}, \mathrm{Ni}\right.$ and $\left.\mathrm{Cr}\right)$.

\begin{tabular}{lcccc}
\hline & & \multicolumn{2}{c}{ Activation energy } & \\
$\mathbf{M}^{\prime}$ & $\begin{array}{c}\text { Lattice parameter } \\
\mathrm{a}(\AA)\end{array}$ & $E_{\mathrm{a}}(\mathrm{eV})$ & $E_{\mathrm{b}}(\mathrm{eV})$ & $\begin{array}{c}\text { Hopping energy } \\
W_{\mathbf{H}}(\mathrm{eV})\end{array}$ \\
\hline $\mathrm{Co}$ & 3.875 & 0.39 & 0.56 & 0.18 \\
$\mathrm{Ni}$ & 3.888 & 0.21 & 0.54 & 0.32 \\
$\mathrm{Cr}$ & 3.892 & 0.18 & 0.43 & 0.25 \\
\hline
\end{tabular}

dried powders were calcined at $1523 \mathrm{~K}$. The calcined and reground powders were pelletized in the form of cylindrical pellets. These pellets were sintered at $1673 \mathrm{~K}$ for $12 \mathrm{~h}$ and then furnace cooled. X-ray diffraction patterns of the final products were taken in a X-ray diffractometer using $\mathrm{Cu} \mathrm{K}_{\alpha}$ radiation. For conductivity measurements, polished pellets were coated with silver paint cured at $900 \mathrm{~K}$ for $30 \mathrm{~min}$. Conductance $(G=1 / R)$ was measured as a function of temperature and frequency using Hewlett Packard impedance analyser, HP 4192A LF.

\section{Results and discussion}

Powder X-ray diffraction data of the samples show the formation of single phase material. XRD patterns did not contain any line characteristic of the constituent oxides or any other compound among them. XRD data could be indexed on the basis of a cubic unit cell similar to strontium titanate and calculated lattice parameter ' $a$ ' is given in table 1 . It is noted from the table that the lattice parameter is highest for $\mathrm{Cr}$ substituted samples and lowest for Co substituted samples. This can be understood in terms of decreasing ionic radii of the substituents $\{\mathrm{La}(1.32 \AA), \operatorname{Co}(0.525 \AA), \mathrm{Ni}(0.56 \AA)$, $\operatorname{Cr}(0.615 \AA)\}$ in comparison to ions going to be substituted $\{\operatorname{Sr}(1.44 \AA), \operatorname{Ti}(0.605 \AA)\}$ (Shannon and Prewitt 1970).

For all the samples, the variation of $\log \sigma_{\mathrm{AC}}$ as a function of $\log f$ at two different temperatures is shown in figure 1 for all the samples at two different temperatures. It is observed that AC conductivity increases with increase in frequency. Variation of $\log$ $\sigma_{\mathrm{AC}}$ with $\log f$ obeys the relation (Austin and Mott 1969):

$$
\log \sigma_{\mathrm{AC}}=A \omega^{s}
$$

where $A$ is constant at particular temperature and $s$ a weak function of frequency. In these plots, two regions are observed. A low frequency region where frequency dependence of log of $\mathrm{AC}$ conductivity is less in comparison to higher frequency region. At higher temperatures, frequency dependence of $\mathrm{AC}$ conductivity decreases i.e. the value of $s$ decreases. A variation of logarithm of DC resistivity with inverse of temperature for these samples is shown in figure 2 where $\mathrm{DC}$ resistivity was calculated from the slopes of the $V-I$ plots of these samples at different temperatures. Figure 3 shows the variation of $\log$ of $\mathrm{AC}$ conductivity with $1000 / \mathrm{T}$ at $100 \mathrm{KHz}$ for $\mathrm{Co}, \mathrm{Ni}$ and Cr substituted samples. In both figures 2 and 3, two regions are observed. A lower temperature region having low value of activation energy and a higher temperature 
Electrical conduction in $S r_{0.90} L a_{0 \cdot 10} T i_{0.90} M_{0 \cdot 10}^{\prime} O_{3}$

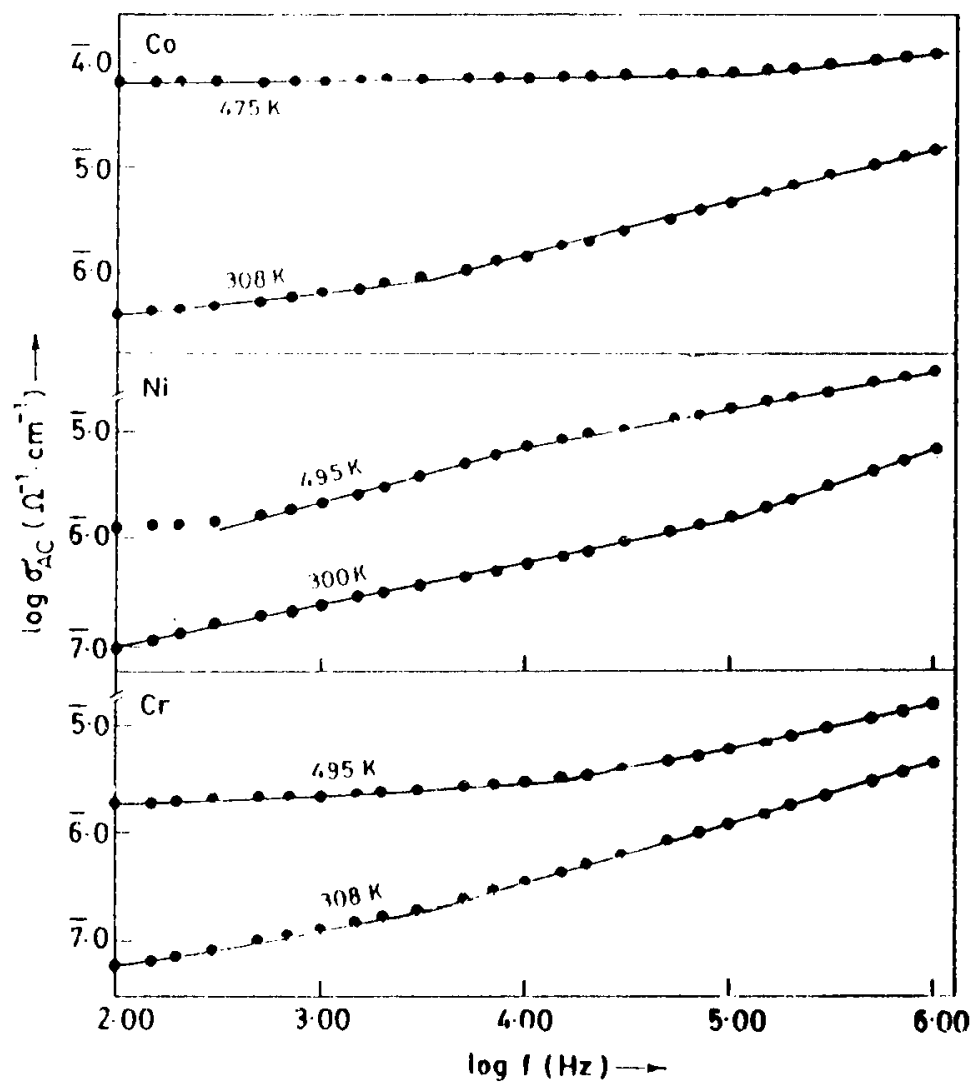

Figure 1. Variation of $\log \sigma_{\mathrm{Ac}}$ as a function of frequency at two different temperatures.

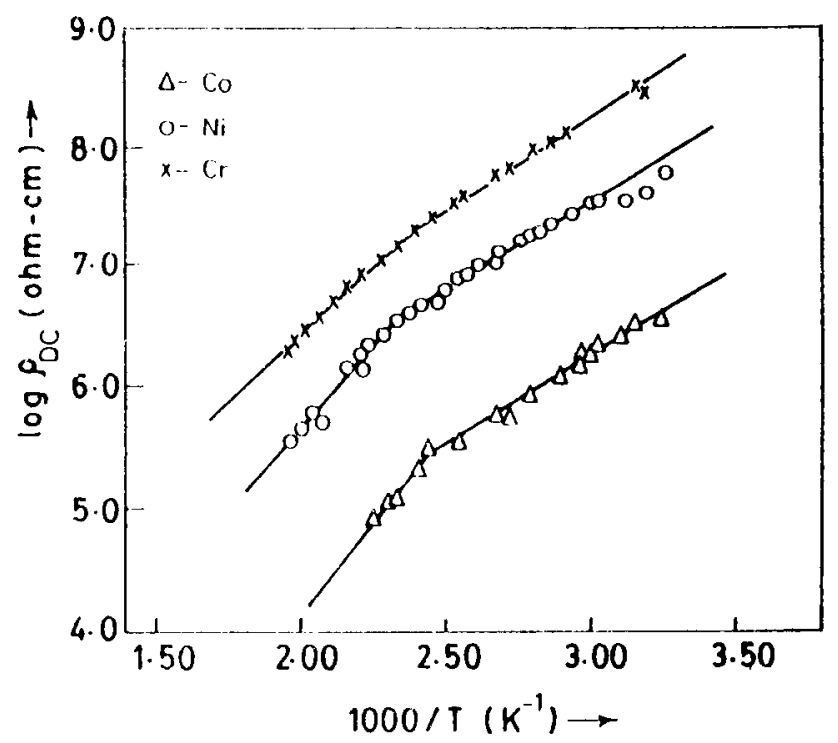

Figure 2. Plots of $\log \rho_{\mathrm{DC}}$ against inverse of temperature. 


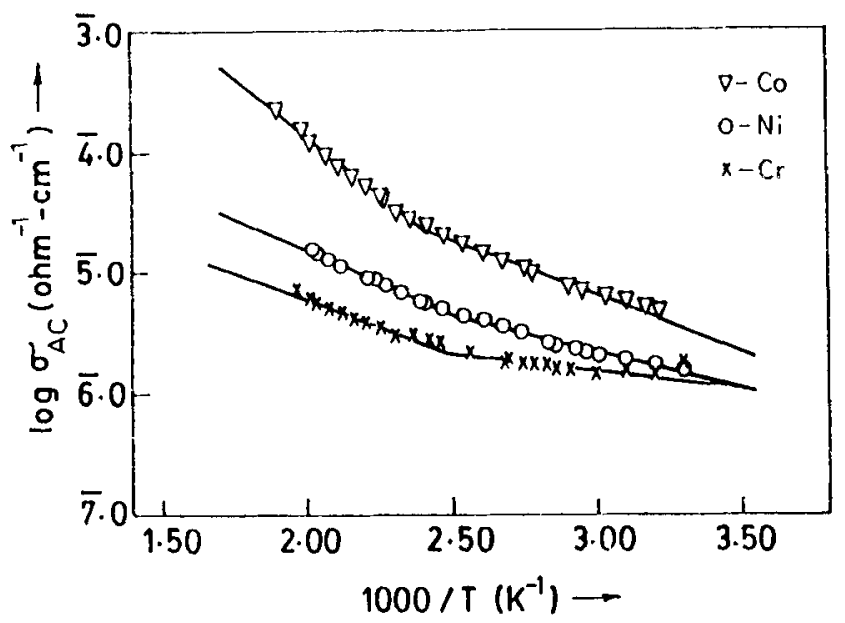

Figure 3. Variation of $\log \sigma_{\mathrm{AC}}$ as a function of inverse of temperatures for different compositions.

region where dependence of $\mathrm{AC}$ conductivity on temperature is more pronounced. Activation energies calculated from these plots using least square fit of the data are given in table 1 for both temperature regions.

For these compositions, the conduction process is predominantly due to the $3 \mathrm{~d}$ electrons of the transition metal ions as already reported (Parkash et al 1993a,b). In lower temperature region, the conductivity may be due to hopping of charge carriers among localized sites (Pollak and Gaballe 1961; Austin and Mott 1969) giving rise to a frequency dependence of $\mathrm{AC}$ conductivity as $\omega^{s}$ and little temperature dependence. At higher temperatures, the conduction occurs by transport of charge carriers excited into localized states at the valence band edge and hopping at energies close to it. The frequency and temperature dependence of conductivity in such a case is given by (Elliot 1978)

$$
\sigma_{\mathrm{AC}}(\omega, T)=(\pi / 96)^{3} e^{2} \alpha^{-5} \cdot k T\left[N\left(E_{\mathrm{A}}\right)\right]^{2} \omega\left[\ln \left(v_{\mathrm{ph}} / \omega\right)\right]^{4} \exp \left[-\left(E_{\mathrm{F}}-E_{\mathrm{A}}\right) / k T\right] .
$$

The variation of DC resistivity for this mechanism is given by

$$
\rho=\rho_{0} \exp \left\{\left(E_{\mathrm{F}}-E_{\mathrm{A}}-W_{\mathrm{H}}\right) / k T\right\},
$$

where $E_{\mathrm{A}}$ represents the valence band edge and $W_{\mathrm{H}}$ the activation energy for hopping. For this mechanism both $\mathrm{AC}$ and $\mathrm{DC}$ conductivity vary exponentially with temperature. However, the activation energies for AC and DC conduction are different. The difference between these two values gives the value of activation for hopping. The value of hopping energy $\left(W_{\mathrm{H}}\right)$ for these compositions are given in table 1. Same conduction mechanism is operative in similar systems already reported (Parkash et al 1993a, b).

In this system, conductivity increases from $\mathrm{Cr}$ substituted composition to Co substituted composition. In these samples, transition metal ions of different valencies are present. Presence of these multivalent ions can be understood as follows. When these materials are sintered at high temperature, they lose traces of oxygen during sintering and in this process extra electrons are released. These electrons are captured by transition metal ions and produce small amounts of ions of different valency. These 
ions which are on $\mathrm{Ti}^{4+}$ sites form dipole with positively charged oxygen vacancies $\mathrm{V}_{\mathrm{o}}$. These dipoles can change their orientation by hopping of electrons among these transition metal ions of different valencies. As the radii of transition metal ions decreases, change in orientation of dipoles (hopping of electrons) become easy due to change in polarizability. For charge transport in these samples. transition metal ion and oxygen vacancy distance is important. As the ionic radii of transition metal ion decreases, the transition metal ion-oxygen vacancy distance increases which facilitate the hopping of charge carriers (George and Grace 1969). In transition metal ions, the ability of ions to change the valency in different environment also decreases from Co to $\mathrm{Cr}$ ions. Measurement of magnetic susceptibility of $\mathrm{Cr}, \mathrm{Ni}$ and $\mathrm{Co}$ doped $\mathrm{BaTiO}_{3}$ compositions also show the increasing trend from $\mathrm{Cr}$ substituted samples to $\mathrm{Co}$ substituted compositions (Ihrig 1978).

\section{Acknowledgements}

Authors are thankful to the All India Council for Technical Education, New Delhi for financial assistance and one of us (HST) is very much thankful to Prof. V B Tare and Dr Om Parkash, Department of Metallurgical Engineering, Banaras Hindu University, Varanasi for facilities.

\section{References}

Austin I G and Mott N F 1969 Adu. Phys. 1841

Buchanan R C 1986 Ceramic materials for electronics (New York: Marcel Dekker)

Elliott S R 1978 Philos. Mag. 37553

George W L and Grace R E 1969 J. Phys. Chem. Solids 30881

Ihrig H 1978 J. Phys. C: Solid State Phys. 11819

Om Parkash, Prasad C D and Kumar D 1987 J. Solid State Chem. 69385

Om Parkash. Prasad C D and Kumar D 1989a J. Mater. S. $\cdot$. Letts. 8475

Om Parkash. Prasad C D and Kumar D 1989 b Phys. Status Solidi A116 k81

Om Parkash. Prasad C D and Kumar D 1990 J. Mater. Sci. 25487

Om Parkash. Tewari H S. Tare V B and Kumar D 1993a J. Alloys \& Compounds 190243

Om Parkash, Tewari H S. Tare V B and Kumar D 1993b J. Phys. D: Appl. Phys. 26676

Pollak M and Gaballe T N 1961 Phys. Rev. 1221742

Shannon R D and Prewitt C T 1970 Acta Crystallogr. B26 1046 